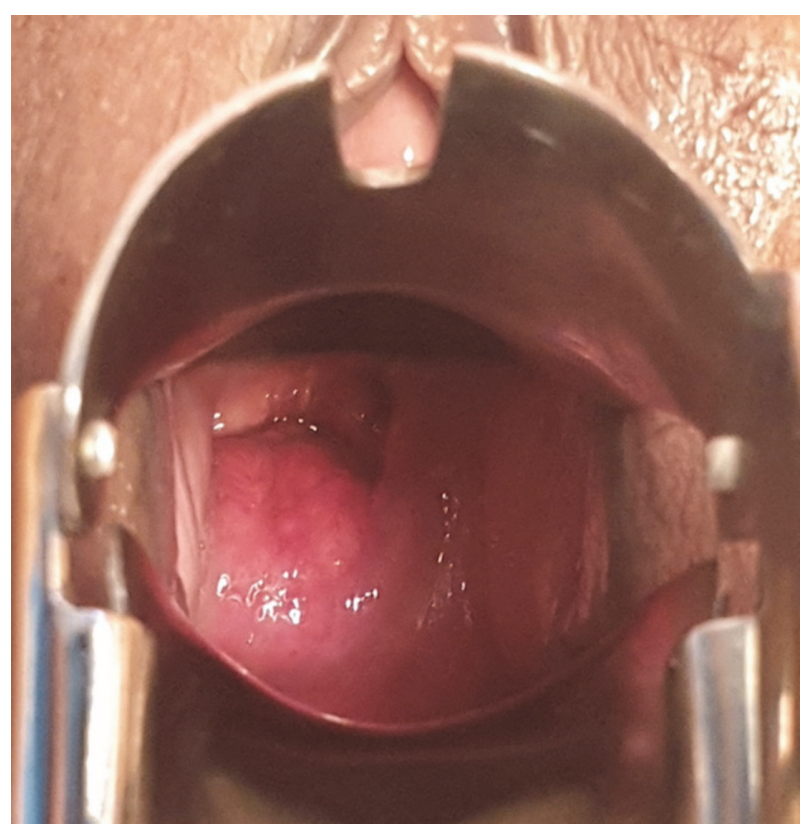

Abstract EPV051/\#264 Figure 2

immunohistochemistry were consistent with SCNC. Imaging studies were done to determine the extent of the tumor. A diagnosis of small cell neuroendocrine carcinoma of the cervix, stage IIIC1r with pelvic organ prolapse, stage IVC was made. Uterine procidentia was reduced using a gellhorn pessary. The patient received external beam radiation therapy followed by brachytherapy, and chemotherapy with Cisplatin and Etoposide.

Results There was marked reduction of the cervical mass, and complete resolution of the pelvic organ prolapse, as well.

Conclusions This is the first report of small cell neuroendocrine carcinoma of the cervix complicated with uterine procidentia, locally and internationally. It required a multidisciplinary approach involving a urogynecologist, a gynecologic oncologist, and a radiation oncologist. Standard treatment guidelines for this rare tumor and case are yet to be established.

\section{EPV052/\#273 A NOVEL IMAGE-GUIDED POINT-OF-CARE ETHYL CELLULOSE ETHANOL ABLATION STRATEGY FOR RECURRENT LOCALIZED CERVICAL CANCER}

1,2,3 J Agudogo ${ }^{*},{ }^{2} \mathrm{C}$ Nief, ${ }^{2} \mathrm{~A}$ Gonzales, ${ }^{4} \mathrm{~J}$ Mueller, ${ }^{2} \mathrm{~B}$ Crouch, ${ }^{5} \mathrm{R}$ Previs, ${ }^{2} \mathrm{~N}$ Ramanujam. ${ }^{1}$ Duke University, School of Medicine, Durham, USA; ${ }^{2}$ Duke University, Biomedical Engineering, Durham, USA; ${ }^{3}$ Duke University, Center For Global Women's Health Technologies, Durham, USA; ${ }^{4}$ University of Maryland, School of Engineering, College Park, USA; ${ }^{5}$ Duke University School of Medicine, Obstetrics and Gynecology, Durham, USA

\subsection{6/ijgc-2021-IGCS.120}

Objectives Local ablation is a promising option for recurrent localized cervical cancer in non-surgical candidates who fail platinum-based chemotherapy and radiation. We developed a low-cost polymer-assisted ethanol ablative therapy, Point-ofcare Ethanol Ethyl Cellulose (PEEC), that overcomes the main shortcoming of ethanol ablation: off-target ethanol leakage. Since increased tumor coverage of ablative therapies results in reduced tumor progression and improved clinical outcomes, we hypothesized that PEEC with image-guidance would optimize cervicovaginal tumor coverage resulting in decreased tumor progression and off-target effects.

Methods A syngeneic cervicovaginal tumor model was established in C57BL/6 mice using TC1-Luc, HPV16 E6/E7+ cells expressing luciferase. Mice were randomized into imageguided PEEC (IG-PEEC), PEEC without image guidance (PEEC only), and saline ablation groups $(n=5)$. Tumors were monitored with bioluminescence imaging via a Perkin-Elmer in vivo imaging system (IVIS) and calipers. Ablations consisted of two intratumoral injections $(50 \mathrm{~mL}$ each) of either PEEC or saline. Image-guided ablations were performed using IVIS to both target PEEC injections at regions of highest radiance intensity (correlated to tumor mass) and to assess tumor coverage.

Results Tumors treated with IG-PEEC performed best with lower total radiance, volumes and weights, and longer survival compared to PEEC only and saline groups $(\mathrm{p}<0.05)$; both PEEC groups demonstrated reduced tumor growth compared to saline $(\mathrm{p}<0.05)$. off-target damage (ulceration) rates were lower for the IG-PEEC $(n=0,0 \%)$ versus the PEEC only $(n=2,40 \%)$ group.

Conclusions PEEC ablation enhanced by image-guidance significantly controls HPV16 E6/E7+ cervicovaginal tumor progression. This supports image-guidance as a critical component in optimizing PEEC ablation and eventual clinical translation.

\section{EPV053/\#282 OBSTETRICAL AND ONCOLOGIC OUTCOMES AFTER ABDOMINAL RADICAL TRACHELECTOMY}

ME Capilna*. 'George Emil Palade' University of Medicine, Pharmacy, Science and Technology, First Obstetrics and Gynecology Clinic, Targu Mures, Romania

\subsection{6/ijgc-2021-IGCS.121}

Objectives To describe retrospectively our experience following abdominal radical trachelectomy (ART), including 5 performed during pregnancy, in terms of complications, obstetrical and oncologic outcomes.

Methods Between 2010 and 2020, all patients with early stage cervical cancer deserving to preserve their fertility were considered for ART. Out of the 19 patients who have met the inclusion criteria for ART, in 18 the trachelectomy was performed and only 1 case needed conversion to radical hysterectomy.

Results Patients' mean age was 31 years old (range 24- 38); two thirds of them were nulliparous. Six women $(33.33 \%)$ were staged as IA2, $4(22.22 \%)$ IB1, 5 (27.78\%) IB2, and 4 (22.22\%) stage IB3 disease. Only one intraoperative complication has occurred - both bladder and right ureteral injuries. Early postoperative complications were urinary bladder dysfunction (33.33\%), symptomatic pelvic lymphocele which was drained (11.1\%), peritonitis (5.5\%), and wound infection (5.5\%). Late postoperative complications included cervical stenosis (5.5\%), amenorrhea (11.1\%), and pelvic abscess $(5.5 \%)$. Four out of the 18 patients were operated during pregnancy between 14 and 20 weeks; 2 of them have delivered at term, and 2 of them have aborted shortly after the surgery. One patients was operated immediately after caesarean section. Two vaginal recurrences were recorded; and both have been managed by hysterectomy, partial colpectomy and adjuvant chemoradiotherapy. At this moment, all patients are alive with no evidence of disease and 3 of them managed to conceive. 